\title{
Derivation of the Local Lorentz Gauge Transformation of a Dirac Spinor Field in Quantum Einstein-Cartan Theory
}

\author{
Rainer W. Kühne \\ Tuckermannstr. 35, 38118 Braunschweig, Germany \\ e-mail: kuehne70@gmx.de
}

July 2, 2018

\begin{abstract}
I examine the groups which underly classical mechanics, non-relativistic quantum mechanics, special relativity, relativistic quantum mechanics, quantum electrodynamics, quantum flavourdynamics, quantum chromodynamics, and general relativity. This examination includes the rotations $S O(2)$ and $S O(3)$, the Pauli algebra, the Lorentz transformations, the Dirac algebra, and the $U(1), S U(2)$, and $S U(3)$ gauge transformations. I argue that general relativity must be generalized to Einstein-Cartan theory, so that Dirac spinors can be described within the framework of gravitation theory.
\end{abstract}

\section{Keywords}

Einstein-Cartan theory, quantum gravity, energy-momentum tensor, Lorentz transformations, gauge invariance, quantum field theories

\section{Introduction}

I will present my argumentation in a didactic way. So I will start at a very elementary level. If not denoted otherwise then Latin indices run from 1 to 3 , Greek indices run from 0 to 3 . I will use the Einstein summation convention, where it is summed over all indices which appear twice. Moreover I will use the natural units

$$
\hbar=c=\varepsilon_{0}=1
$$

where $\hbar=h / 2 \pi$ denotes the reduced Planck constant, $c$ the speed of light, and $\varepsilon_{0}$ the electric field constant. Inner indices of matrices will be dropped.

Readers who would like to learn more about the theories which will be mentioned in this paper are recommended to the comprehensive works of Goldstein [1] (classical mechanics), Jackson [2] (classical electrodynamics), Kilmister [3] (special relativity), Weinberg [4] and Misner, Thorne and Wheeler [5] (general relativity),
Hehl et al. [6] (Einstein-Cartan theory), Messiah [7] (non-relativistic quantum mechanics), Bjorken and Drell [8] (relativistic quantum mechanics), Bjorken and Drell [9] (quantum electrodynamics), Taylor [10] (quantum flavourdynamics), Politzer [11] and Marciano and Pagels [12] (quantum chromodynamics), and Kühne [13] (quantum electromagnetodynamics).

\section{Classical Mechanics}

The space of classical mechanics is described by the three-dimensional Euclidian space. The scalar product of the three-vectors $a_{i}$ and $b_{i}$ is given by

$$
a \cdot b=\delta_{i j} a_{i} b_{j}
$$

where

$$
\delta_{i j}=\operatorname{diag}(1,1,1)
$$

denotes the Kronecker symbol. The vector product is given by

$$
(a \times b)_{k}=\varepsilon_{i j k} a_{i} b_{j}
$$

where $\varepsilon_{i j k}$ denotes the totally anti-symmetric Levi-Civita symbol. The square of the infinitesimal line element is given by

$$
d s^{2}=\delta_{i j} d x_{i} d x_{j}
$$

where $d x_{i}$ denotes the infinitesimal coordinate difference of the two space-points $x_{i}$ and $y_{i}$

$$
d x_{i}=\lim _{y_{i} \rightarrow x_{i}}\left(y_{i}-x_{i}\right)
$$

A rotation $R$ around the rotation angle $\varphi$ in the two-dimensional subspace is described by the orthogonal rotation group $S O(2)$

$$
\begin{aligned}
R & =\exp (-i \varphi D) \\
& =\left(\begin{array}{cc}
\cos \varphi & -\sin \varphi \\
\sin \varphi & \cos \varphi
\end{array}\right) \in S O(2)
\end{aligned}
$$

where, because of the Euler equation

$$
e^{-i \varphi}=\cos \varphi-i \sin \varphi
$$


the generator of the rotation is

$$
D=\left(\begin{array}{cc}
0 & -i \\
i & 0
\end{array}\right)
$$

A rotation $R$ around the rotation angle $\varphi_{i}$ in the three-dimensional space is described by the rotation group $S O(3)$

$$
R=\exp \left(-i \varphi_{i} D_{i}\right) \in S O(3)
$$

where the generators $D_{i}$ of the rotation satisfy the commutator relation

$$
\left[D_{i}, D_{j}\right]=i \varepsilon_{i j k} D_{k}
$$

A representation of the generators of the $S O(3)$ group is

$$
\begin{aligned}
D_{1} & =\left(\begin{array}{ccc}
0 & 0 & 0 \\
0 & 0 & -i \\
0 & i & 0
\end{array}\right) \\
D_{2} & =\left(\begin{array}{ccc}
0 & 0 & i \\
0 & 0 & 0 \\
-i & 0 & 0
\end{array}\right) \\
D_{3} & =\left(\begin{array}{ccc}
0 & -i & 0 \\
i & 0 & 0 \\
0 & 0 & 0
\end{array}\right)
\end{aligned}
$$

Note that the number of generators of the orthogonal groups is given by

$$
\operatorname{dim} S O(n)=n(n-1) / 2
$$

and that the generators of $S O(n)$ are Hermitean.

\section{Non-Relativistic Quantum Mechanics}

Classical angular momentum is continuous. In quantum physics orbital angular momentum is quantized in units of $\hbar$ and intrinsic spin is quantized in units of $\hbar / 2$. Intrinsic spin is described by the unitary group $S U(2)$. Its generators are the three Pauli matrices $\sigma_{i}$. An often used representation of the Pauli matrices is

$$
\begin{aligned}
\sigma_{1} & =\left(\begin{array}{ll}
0 & 1 \\
1 & 0
\end{array}\right) \\
\sigma_{2} & =\left(\begin{array}{cc}
0 & -i \\
i & 0
\end{array}\right) \\
\sigma_{3} & =\left(\begin{array}{cc}
1 & 0 \\
0 & -1
\end{array}\right)
\end{aligned}
$$

The commutator of the group $S U(2)$ is given by

$$
\left[\sigma_{i}, \sigma_{j}\right]=2 i \varepsilon_{i j k} \sigma_{k}
$$

The groups $S U(2)$ and $S O(3)$ are local isomorphic for angles $0 \leq \varphi<2 \pi$ where the group
$S U(2)$ is covered by the group $S O(3)$. Note also that the commutators eq. (11) and eq. (19) differ by a factor of two. The anti-commutator is

$$
\left\{\sigma_{i}, \sigma_{j}\right\}=2 \delta_{i j}
$$

The multiplication of two three-vectors $a_{i}$ and $b_{i}$ is given by

$$
\begin{aligned}
(\sigma \cdot a)(\sigma \cdot b) & =\left(\sigma_{i} \cdot a_{i}\right)\left(\sigma_{j} \cdot b_{j}\right) \\
& =\left(\delta_{i j}+i \varepsilon_{i j k} \sigma_{k}\right) a_{i} b_{j} \\
& =a \cdot b+i \sigma \cdot(a \times b)
\end{aligned}
$$

According to non-relativistic quantum mechanics both spin and isospin are invariant under global transformations of the group $S U(2)$. If $\Psi$ denotes a two-component Pauli-spinor, $\varphi_{i}$ the three-component rotation angle vector, $\sigma_{i}$ the Pauli matrices, and $x$ a space-time point, then

$$
\Psi \prime(x)=\exp \left(-i \varphi_{i} \sigma_{i} / 2\right) \Psi(x)
$$

If $\Psi$ denotes a two-component iso-spinor, $\varphi_{i}$ the three-component phase vector, and $\tau_{i}$ the Pauli matrices, then

$$
\Psi \prime(x)=\exp \left(-i \varphi_{i} \tau_{i} / 2\right) \Psi(x)
$$

\section{Special Relativity}

The special theory of relativity is invariant under the semi-simple Poincare group. The parameters of its translational part are time and three-position. The parameters of its rotational part are the rotation angle three-vector and the three-component Lorentz boost.

The scalar product of the two four-vectors $a^{\mu}$ and $b^{\mu}$ is given by

$$
a \cdot b=g_{\mu \nu} a^{\mu} b^{\nu}
$$

where $g_{\mu \nu}$ denotes the metric tensor. It is

$$
\begin{aligned}
& g^{\mu \nu}=g_{\mu \nu} \\
& g^{\mu}{ }_{\nu}=g_{\nu}{ }^{\mu}=\delta_{\nu}^{\mu}=\operatorname{diag}(1,1,1,1)
\end{aligned}
$$

In Minkowski coordinates the metric tensor is represented by

$$
g_{\mu \nu}=\operatorname{diag}(1,-1,-1,-1)
$$

The square of the infinitesimal line element is given by

$$
d s^{2}=g_{\mu \nu} d x^{\mu} d x^{\nu}
$$

where $d x^{\mu}$ denotes the infinitesimal coordinate difference of the two space-time points $x^{\mu}$ and $y^{\mu}$

$$
d x^{\mu}=\lim _{y^{\mu} \rightarrow x^{\mu}}\left(y^{\mu}-x^{\mu}\right)
$$


A rotation around the $z$-axis by the rotation angle $\varphi$ is given by

$$
a^{\mu}{ }_{\nu}=\left(\begin{array}{cccc}
1 & 0 & 0 & 0 \\
0 & \cos \varphi & -\sin \varphi & 0 \\
0 & \sin \varphi & \cos \varphi & 0 \\
0 & 0 & 0 & 1
\end{array}\right)
$$

A Lorentz boost along the $x$-axis by the speed $v$ is given by

$$
a_{\nu}^{\mu}=\left(\begin{array}{cccc}
\gamma & -\beta \gamma & 0 & 0 \\
-\beta \gamma & \gamma & 0 & 0 \\
0 & 0 & 1 & 0 \\
0 & 0 & 0 & 1
\end{array}\right)
$$

where

$$
\begin{aligned}
& \beta=v / c \\
& \gamma=1 / \sqrt{1-v^{2} / c^{2}}
\end{aligned}
$$

In general, a Lorentz transformation $a^{\mu}{ }_{\nu}$ of a four-position $x^{\mu}$ is given by

$$
x^{\mu}=a^{\mu}{ }_{\nu} x^{\nu}
$$

The Lorentz transformation of a four-derivative $\partial_{\mu}=\partial / \partial x^{\mu}$ is given by

$$
\partial \prime_{\mu}=a_{\mu}{ }^{\nu} \partial_{\nu}
$$

Finally, a Lorentz transformation around the parameter $\omega_{\alpha \beta}$ is given by

$$
a_{\nu}^{\mu}=\exp \left(\frac{1}{2} \omega_{\alpha \beta} I^{\alpha \beta}\right)_{\nu}^{\mu} \in O(1,3)
$$

where a representation of the six generators of the Lorentz group $S O(1,3)$ is

$$
\begin{aligned}
& I^{10}=\left(\begin{array}{cccc}
0 & -1 & 0 & 0 \\
1 & 0 & 0 & 0 \\
0 & 0 & 0 & 0 \\
0 & 0 & 0 & 0
\end{array}\right) \\
& I^{20}=\left(\begin{array}{cccc}
0 & 0 & -1 & 0 \\
0 & 0 & 0 & 0 \\
1 & 0 & 0 & 0 \\
0 & 0 & 0 & 0
\end{array}\right) \\
& I^{30}=\left(\begin{array}{cccc}
0 & 0 & 0 & -1 \\
0 & 0 & 0 & 0 \\
0 & 0 & 0 & 0 \\
1 & 0 & 0 & 0
\end{array}\right) \\
& I^{13}=\left(\begin{array}{cccc}
0 & 0 & 0 & 0 \\
0 & 0 & 0 & -1 \\
0 & 0 & 0 & 0 \\
0 & 1 & 0 & 0
\end{array}\right) \\
& I^{23}=\left(\begin{array}{cccc}
0 & 0 & 0 & 0 \\
0 & 0 & 0 & 0 \\
0 & 0 & 0 & -1 \\
0 & 0 & 1 & 0
\end{array}\right) \\
& I^{12}=\left(\begin{array}{cccc}
0 & 0 & 0 & 0 \\
0 & 0 & -1 & 0 \\
0 & 1 & 0 & 0 \\
0 & 0 & 0 & 0
\end{array}\right)
\end{aligned}
$$

\section{Relativistic Quantum Me- chanics}

The group which underlies the kinematics of relativistic quantum mechanics and relativistic quantum field theory is the Poincare group. Isospin is invariant under global transformations of the group $S U(2)$. The generators are the three Pauli matrices $\tau_{i}$. The three Pauli matrices $\sigma_{i}$ of spin are generalized by the four Dirac matrices $\gamma_{\mu}$. An often used representation of the Dirac matrices is

$$
\begin{aligned}
\gamma_{0} & =\left(\begin{array}{cc}
1 & 0 \\
0 & -1
\end{array}\right) \\
\gamma_{i} & =\left(\begin{array}{cc}
0 & \sigma_{i} \\
-\sigma_{i} & 0
\end{array}\right)
\end{aligned}
$$

It is

$$
\begin{aligned}
& \gamma^{0}=\gamma_{0} \\
& \gamma^{i}=-\gamma_{i}
\end{aligned}
$$

The commutator is

$$
\left[\gamma_{\mu}, \gamma_{\nu}\right]=-2 i \sigma_{\mu \nu}
$$

which is the definition of the generalized Dirac matrices $\sigma_{\mu \nu}$. The anti-commutator gives

$$
\left\{\gamma_{\mu}, \gamma_{\nu}\right\}=2 g_{\mu \nu}
$$

By using the representation eqs. (43) and (44) of the Dirac matrices and the representation eqs. (16), (17) and (18) of the Pauli matrices, the anti-commutator gives the representation eq. (27) of the metric tensor in Minkowski coordinates. Moreover it is

$$
\gamma^{5}=\gamma_{5}=-i \gamma_{0} \gamma_{1} \gamma_{2} \gamma_{3}
$$

The multiplication of two four-vectors $a^{\mu}$ and $b^{\mu}$ is given by

$$
\begin{aligned}
(\gamma \cdot a)(\gamma \cdot b) & =\left(\gamma_{\mu} a^{\mu}\right)\left(\gamma_{\nu} b^{\nu}\right) \\
& =\left(g_{\mu \nu}-i \sigma_{\mu \nu}\right) a^{\mu} b^{\nu}
\end{aligned}
$$

The Lorentz transformation of a four-component spin $1 / 2$ Dirac spinor field $\Psi(x)$ is given by

$$
\Psi \prime(x \prime)=\exp \left(-\frac{i}{4} \sigma_{\mu \nu}\left(\frac{1}{2} \omega_{\alpha \beta} I^{\alpha \beta}\right)^{\mu \nu}\right) \Psi(x)
$$

which is a generalization of eq. (36) which considers both the Lorentz transformation of the elementary particle and its intrinsic spin.

\section{Relativistic Field Theory}

Quantum electrodynamics is invariant under local transformations of the gauge group $U(1)$. 
If $\Psi$ describes a four-component Dirac spinor, $x$ a space-time point, $e$ the elementary electric charge, $\varphi$ the gauge phase, $D_{\mu}$ the covariant derivative, $\partial_{\mu}$ the partial four-derivative, and $A^{\mu}$ the electromagnetic four-potential, then the gauge transformation is

$$
\begin{aligned}
\Psi \prime(x) & =\exp (-i e \varphi(x)) \Psi(x) \\
i D_{\mu} & =i \partial_{\mu}-e A_{\mu}(x) \\
A^{\prime}{ }_{\mu}(x) & =A_{\mu}(x)-\partial_{\mu} \varphi(x)
\end{aligned}
$$

Note that $U(1)$ and $S O(2)$ are isomorphic.

Quantum flavourdynamics is invariant under local transformations of the gauge group $S U(2) \times U(1)$. If $\Psi$ denotes an eight-component iso-spinor Dirac spinor, $x$ a space-time point, $g$ the weak coupling constant, $\varphi_{i}$ the threecomponent gauge phase isovector, $\tau_{i}$ the Pauli matrices, and $W_{\mu}^{i}$ the weak isovector fourpotentials, then the gauge transformations of the $S U(2)$ part are

$$
\begin{aligned}
\Psi \prime(x)= & \exp \left(-i g \varphi_{i}(x) \tau_{i} / 2\right) \Psi(x) \\
i D_{\mu}= & i \partial_{\mu}-\frac{g}{2} W_{\mu}^{i}(x) \tau_{i} \\
W \prime_{i}^{\mu}(x)= & W_{i}^{\mu}(x)-\partial^{\mu} \varphi_{i}(x) \\
& -g \varepsilon_{i j k} \varphi_{j}(x) W_{k}^{\mu}(x)
\end{aligned}
$$

Quantum chromodynamics is invariant under local transformations of the gauge group $S U(3)$. If $\Psi$ denotes a twelve-component colourvector Dirac spinor, $x$ a space-time point, $g$ the strong coupling constant, $\varphi_{i}$ the eightcomponent gauge phase vector, $\lambda_{i}$ the eight GellMann matrices, and $G_{\mu}^{i}$ the eight gluon fourpotentials, then the gauge transformations are

$$
\begin{aligned}
\Psi \prime(x)= & \exp \left(-i g \varphi_{i}(x) \lambda_{i} / 2\right) \Psi(x) \\
i D_{\mu}= & i \partial_{\mu}-\frac{g}{2} G_{\mu}^{i}(x) \lambda_{i} \\
G \prime_{i}^{\mu}(x)= & G_{i}^{\mu}(x)-\partial^{\mu} \varphi_{i}(x) \\
& -g f_{i j k} \varphi_{j}(x) G_{k}^{\mu}(x)
\end{aligned}
$$

where the indices $i, j, k$ run from 1 to 8 .
A representation of the Gell-Mann matrices is

$$
\begin{aligned}
& \lambda_{1}=\left(\begin{array}{lll}
0 & 1 & 0 \\
1 & 0 & 0 \\
0 & 0 & 0
\end{array}\right) \\
& \lambda_{2}=\left(\begin{array}{ccc}
0 & -i & 0 \\
i & 0 & 0 \\
0 & 0 & 0
\end{array}\right) \\
& \lambda_{3}=\left(\begin{array}{ccc}
1 & 0 & 0 \\
0 & -1 & 0 \\
0 & 0 & 0
\end{array}\right) \\
& \lambda_{4}=\left(\begin{array}{lll}
0 & 0 & 1 \\
0 & 0 & 0 \\
1 & 0 & 0
\end{array}\right) \\
& \lambda_{5}=\left(\begin{array}{ccc}
0 & 0 & -i \\
0 & 0 & 0 \\
i & 0 & 0
\end{array}\right) \\
& \lambda_{6}=\left(\begin{array}{lll}
0 & 0 & 0 \\
0 & 0 & 1 \\
0 & 1 & 0
\end{array}\right) \\
& \lambda_{7}=\left(\begin{array}{ccc}
0 & 0 & 0 \\
0 & 0 & -i \\
0 & i & 0
\end{array}\right) \\
& \lambda_{8}=\frac{1}{\sqrt{3}}\left(\begin{array}{ccc}
1 & 0 & 0 \\
0 & 1 & 0 \\
0 & 0 & -2
\end{array}\right)
\end{aligned}
$$

The commutator of the Gell-Mann matrices is

$$
\left[\lambda_{i}, \lambda_{j}\right]=2 i f_{i j k} \lambda_{k}
$$

and the anti-commutator is

$$
\left\{\lambda_{i}, \lambda_{j}\right\}=\frac{4}{3} \delta_{i j}+2 d_{i j k} \lambda_{k}
$$

Note that the number of the generators of the unitary groups is given by

$$
\operatorname{dim} S U(n)=n^{2}-1
$$

and that the generators of the $S U(n)$ groups are Hermitean.

According to relativistic quantum mechanics and relativistic quantum field theory, the energy-momentum tensor $\Sigma^{\mu \nu}$ of a Dirac spinor $\Psi$ is asymmetric

$$
\begin{aligned}
\Sigma^{\mu \nu}(x)= & -\frac{1}{2}\left(\left(D^{\mu} \bar{\Psi}(x)\right) \gamma^{\nu} \Psi(x)\right) \\
& +\frac{1}{2}\left(\bar{\Psi}(x) \gamma^{\nu} D^{\mu} \Psi(x)\right)
\end{aligned}
$$

where the covariant derivative $D_{\mu}$ is given by the equations (53), (56) and (59).

\section{General Relativity}

The general theory of relativity is invariant under arbitrary curvilinear transformations. It 
is invariant under local transformations of the Lorentz group $S O(1,3)$. The Lorentz boosts depend on the four-position $x^{\mu}$. Note that the Poincare group which underlies special relativity has ten generators, whereas the Lorentz group which underlies general relativity has only six generators. Therefore general relativity is not a generalization of special relativity.

The scalar product of the four-vectors $a^{\mu}$ and $b^{\mu}$ is given by

$$
a \cdot b=g_{\mu \nu}(x) a^{\mu} b^{\nu}
$$

where the metric tensor $g_{\mu \nu}$ depends on the space-time point $x$. The square of the infinitesimal line element is

$$
d s^{2}=g_{\mu \nu}(x) d x^{\mu} d x^{\nu}
$$

The local Lorentz transformation is

$$
a_{\nu}^{\mu}(x)=\exp \left(\frac{1}{2} \omega_{\alpha \beta}(x) I^{\alpha \beta}\right)_{\nu}^{\mu}
$$

which is a generalization of eq. (36).

When a four-vector $C^{\alpha}$ is parallely displaced from the four-position $x^{\mu}$ to the four-position $x^{\mu}+d x^{\mu}$, then it changes according to the prescription

$$
d C^{\alpha}=-\Gamma_{\mu \nu}^{\alpha}(x) C^{\nu} d x^{\mu}
$$

This is the definition of the four-positiondependent affine connection $\Gamma_{\mu \nu}^{\alpha}$. According to general relativity it has only a symmetric part

$$
\{\}_{\mu \nu}^{\alpha}(x)=\frac{1}{2}\left(\Gamma_{\mu \nu}^{\alpha}(x)+\Gamma_{\nu \mu}^{\alpha}(x)\right)
$$

which is named Christoffel symbol. The Riemann curvature tensor is given by

$$
\begin{aligned}
R_{\mu \nu \kappa}^{\lambda}(x)= & \partial_{\kappa} \Gamma_{\mu \nu}^{\lambda}(x)-\partial_{\nu} \Gamma_{\mu \kappa}^{\lambda}(x) \\
& +\Gamma_{\mu \nu}^{\alpha}(x) \Gamma_{\kappa \alpha}^{\lambda}(x)-\Gamma_{\mu \kappa}^{\alpha}(x) \Gamma_{\nu \alpha}^{\lambda}(x)
\end{aligned}
$$

By contraction one gets the Ricci tensor

$$
R_{\mu \nu}(x)=R_{\mu \lambda \nu}^{\lambda}(x)
$$

and the Ricci scalar

$$
R(x)=R_{\mu}^{\mu}(x)
$$

The Einstein field equations are

$$
R_{\mu \nu}(x)-\frac{1}{2} g_{\mu \nu}(x) R(x)=\kappa \Sigma_{\mu \nu}(x)
$$

where

$$
\kappa=-8 \pi G
$$

denotes the Einstein field constant and $\Sigma_{\mu \nu}$ the energy-momentum tensor.
Since the affine connection (Christoffel symbol) is symmetric it follows that the energymomentum tensor of general relativity is symmetric. This is in contrast to the asymmetric energy-momentum tensor of a Dirac spinor of relativistic quantum mechanics. This means that a Dirac spinor cannot be described by the geometry that underlies general relativity.

\section{Einstein-Cartan Theory}

Einstein-Cartan theory is a generalization of general relativity, because within the framework of this theory the anti-symmetric part of the affine connection which is named Cartan's torsion tensor

$$
T_{\mu \nu}^{\alpha}(x)=\frac{1}{2}\left(\Gamma_{\mu \nu}^{\alpha}(x)-\Gamma_{\nu \mu}^{\alpha}(x)\right)
$$

is nonzero. In contrast to the Christoffel symbol the torsion tensor transforms as a tensor under arbitrary curvilinear transformations. Cartan's torsion tensor is related to the spin tensor by

$$
T^{\alpha \mu \nu}(x)=\kappa \tau^{\alpha \mu \nu}(x)
$$

where $\kappa=-8 \pi G$ denotes the Einstein field constant known from general relativity.

The Dirac spinor can be described by the geometry that underlies Einstein-Cartan theory, because this theory describes an asymmetric affine connection and therefore an asymmetric energy-momentum tensor.

Einstein-Cartan theory is invariant under local transformations of the Poincare group. So Einstein-Cartan theory is a generalization of special relativity.

In quantum Einstein-Cartan theory the local Lorentz gauge transformation of a Dirac spinor field $\Psi$ is given by

$$
\Psi \prime(x \prime)=\exp \left(-\frac{i}{4} \sigma_{\mu \nu}\left(\frac{1}{2} \omega_{\alpha \beta}(x) I^{\alpha \beta}\right)^{\mu \nu}\right) \Psi(x)
$$

This equation is a combination of eqs. (51) and (75).

\section{Conclusion}

I argued that the general theory of relativity must be generalized by the Einstein-Cartan theory, because the asymmetric energy-momentum tensor of relativistic quantum mechanics requires the existence of an asymmetric affine connection and therefore the existence of a nonzero torsion tensor. Moreover I pointed out that spin is the source of a gauge field which is associated with Cartan's torsion. This is analogous to 
the situation of isospin which is a pure quantum number in quantum mechanics, but the source of the weak gauge field in quantum flavourdynamics.

\section{References}

[1] H. Goldstein, Classical Mechanics (Addison-Wesley, Reading, 1950).

[2] J. D. Jackson, Classical Electrodynamics (John Wiley and Sons, New York, 1962).

[3] C. W. Kilmister, Special Theory of Relativity (Pergamon Press, Oxford, 1970).

[4] S. Weinberg, Gravitation and Cosmology (John Wiley and Sons, New York, 1972).

[5] C. W. Misner, K. S. Thorne and J. A. Wheeler, Gravitation (Freeman and Company, San Francisco, 1973).

[6] F. W. Hehl, P. von der Heyde, G. D. Kerlick and J. M. Nester, General Relativity with Spin and Torsion: Foundations and Prospects, Reviews of Modern Physics 48, 393-416 (1976).

[7] A. Messiah, Quantum Mechanics (NorthHolland Publishing Company, Amsterdam, 1964).

[8] J. D. Bjorken and S. D. Drell, Relativistic Quantum Mechanics (McGraw-Hill, New York, 1964).

[9] J. D. Bjorken and S. D. Drell, Relativistic Quantum Fields (McGraw-Hill, New York, 1965).

[10] J. C. Taylor, Gauge Theories of Weak Interactions (Cambridge University Press, Cambridge, 1976).

[11] H. D. Politzer, Asymptotic Freedom: An Approach to Strong Interactions, Physics Reports 14, 129-180 (1974).

[12] W. Marciano and H. Pagels, Quantum Chromodynamics, Physics Reports 36, 137276 (1978).

[13] R. W. Kühne, Quantum Field Theory with Electric-Magnetic Duality and Spin-Mass Duality but Without Grand Unification and Supersymmetry, African Review of Physics 6, 165-179 (2011). 